\title{
A probabilistic analysis of argument cogency
}

\section{DAVID GODDEN and FRANK ZENKER}

\author{
Philosophy Department \\ Michigan State University \\ East Lansing, Michigan \\ U.S.A. 48824 \\ Email:dgodden@msu.edu \\ www.davidgodden.ca
}

\author{
Universität Konstanz, Philosophie \\ Postfach D9, 78457 Konstanz, Germany \\ Slovak Academy of Sciences, Institute of Philosophy \\ Klemensova 19, 81364 Bratislava, Slovakia \\ Department of Philosophy \& Cognitive Science \\ LUX, Lund University, Box 192, 22100 Lund, Sweden \\ Email:frank.zenker@fil.lu.se \\ http://www.fil.lu.se/person/FrankZenker
}

Godden, D. and Zenker, F. (2016, forthcoming). A probabilistic analysis of argument cogency. Synthese, vol, pp-pp. doi: 10.1007/s11229-016-1299-2

The final publication is available at Springer via:

http://dx.doi.org/10.1007/s11229-016-1299-2 .

\begin{abstract}
This paper offers a probabilistic treatment of the conditions for argument cogency as endorsed in informal logic: acceptability, relevance, and sufficiency (RSA). Treating a natural language argument as a reason-claim-complex, our analysis identifies content features of defeasible argument on which the RSA conditions depend, namely: (i) change in the commitment to the reason, (ii) the reason's sensitivity and selectivity to the claim, (iii) one's prior commitment to the claim, and (iv) the contextually determined thresholds of acceptability for reasons and for claims. Results contrast with, and may indeed serve to correct, the informal understanding and applications of the RSA criteria concerning their conceptual (in)dependence, their function as update-thresholds, and their status as obligatory rather than permissive norms, but also show how these formal and informal normative approaches can in fact align.
\end{abstract}

KEYWORDS: acceptability; argument appraisal; Bayes Theorem; informal logic; Jeffrey conditionalization; relevance; sufficiency

\section{Introduction}

As John Woods (2000: 15) put it: "Formal logic is a theory of logical forms; and informal logic is all the rest." Informal logicians tend to view "all the rest" as shouldering the real work in the analysis and evaluation of natural language argumentation. Indeed, many reject formal methods. In place of the proof techniques of the truth-functional calculus, typical resources rather include argument diagrams, schemes, and the fallacies. Similarly, rather than endorsing soundness (premise truth and deductive inferential validity) as a standard of good argument, informal logicians speak of cogency (premise acceptability, relevance, and inferential sufficiency). 
In the 1960s, this anti-formalist stance arose in reaction to the only widely available formal apparatus, first-order deductive logic. The breadth of formal resources available today, however, makes a continued disenchantment with them questionable. In fact, their neglect deprives informal logicians of useful resources in appraising defeasible reasoning and argument. Our probabilistic analysis of argument cogency clarifies this core concept of informal logic, provides important correctives to its usual applications, but also yields a sense in which a formal and an informal normative approach align. ${ }^{1}$ Building on groundwork by Oaksford and Hahn (2004) and Korb (2004), among others, this contributes to a burgeoning area of research that successfully applies Bayesian reasoning to natural language argumentation (see Section 2.3), and supplements recent work by Hahn and Hornikx (2016), who show how to formalize argument schemes, like those proposed by Walton, Reed, and Macagno (2008), using a Bayesian approach.

Section 2 identifies the anti-formalist sentiments motivating informal logic, presents cogency as a normative standard for defeasible argument, and briefly surveys recent probabilistic treatments of argumentation. Section 3 introduces the probabilistic calculus, seeking to make its resources more accessible to informal logicians. Section 4 then offers a probabilistic analysis of the informal notions acceptability, relevance, and sufficiency. Section 5 discusses consequences of this analysis. Our conclusions are offered in Section 6.

\section{Background}

\subsection{Informal logic and formal methods}

The development of informal logic is motivated by the pedagogical, the internal, and the empirical critiques of deductive logic as an optimal, or even an apt, tool for the analysis and evaluation of ordinary reasoning and argument (Blair, 2011; Johnson, 2006, Johnson \& Blair, 2002). Since commitments to reasons and claims in argumentative contexts typically remain retractable, informal logicians favor acceptability over truth as an evaluative standard for premises. Similarly, since ordinary reasoning and argument are typically defeasible, and since the quality of inferences typically depends on matters of content rather than form, informal logicians (rightly) reject deductive validity as a standard of inferential goodness (see Hertwig et al., 1997; Evans, 2002).

Informal logicians have rather sought to develop non-formal tools by drawing upon such adjacent fields as applied logic, applied epistemology, cognitive and social psychology, dialogue and communication theory, linguistic pragmatics, dialectics, and rhetoric. As Johnson and Blair state:

By 'informal logic', we mean to designate a branch of logic whose task is to develop non-formal standards, criteria, and procedures for the analysis,

\footnotetext{
${ }^{1}$ Similarly important are the notions of argument strengthening, rebuttal, and counter-rebuttal, which however fall outside the scope of this paper.

${ }^{2}$ Recent work nevertheless applies such formal methods as computer models of defeasible inference to reasoning and argument (e.g., Walton \& Gordon, 2015).

${ }^{3}$ Following the introduction of the RSA criteria by Johnson and Blair in 1977 (Johnson \& Blair, 2006:
} 
interpretation, evaluation, critique and construction of argumentation in everyday discourse. (Johnson \& Blair, 2002: 358; italics added)

Insofar as 'formal' denotes a regulated procedure, Johnson and Blair agree-indeed presuppose - that reasoning and argumentation are rule-governed activities. But they object to formalization being the proper aim in the analysis and appraisal of natural language reasoning and argument (2002: $358 \mathrm{ff}$.), and had at least initially adopted a "wait-and-see" approach regarding the applicability of formal methods.

There is nothing incompatible between the aims of informal logic, properly understood, and the attempt to identify formal structures [e.g.,] in the so-called informal fallacies. In calling for no more precision than the subject matter allows, we do not abandon the demand for as much precision as the subject matter allows. [...] Informal logic is not opposed to formal analysis; it is opposed to the mistaken view that the [proper] subject matter of formal deductive logic is [natural language] argument. (Blair \& Johnson, 1987: 148)

As the informal logic program developed, however, it was increasingly characterized as a reaction to the use of formal methods in the study of reasoning and argument: "informal logic is informal because it abandons the notion of logical form as the key to understanding the structure of argument. ... What we reject is the view that (with rare exceptions) the salient criteria for evaluating arguments are a function of their logical form" (Johnson \& Blair, 2002: 359). Describing the eventual maturation of their program, Johnson (2011) states: "As these projects [of informal logic] were pursued, it became ever clearer that we were involved in a logic (taking the central task of logic to be the development of norms for reasoning) that was irretrievably informal' (2011: 29; emphasis added). ${ }^{2}$

\subsection{Cogency}

In place of soundness, informal logic offers cogency as the standard of good argument, where 'cogent' broadly means well-reasoned: a cogent argument meets a situationally appropriate standard of reason-giving that can be variously explained (e.g., epistemically, dialectically, virtue-theoretically, etc). Viewing arguments as abstract inferential objects whose instances arguers transact in acts of arguing, product-centric approaches treat cogency as a normative property of argument-products. Of course, dialectical or rhetorical considerations bear on the evaluation of such acts. Nevertheless, arguing well centrally involves deploying good arguments, and good arguers do this well.

Informal logicians broadly agree that the goodness of an argument is a function of, on one hand, the adequacy of reasons (premises), and the quality of the inferential link between reasons and claim (conclusion), on the other. This gives rise to the relevance, sufficiency, acceptability (RSA) account of cogency (Johnson \& Blair, 2002: 369ff.;

\footnotetext{
${ }^{2}$ Recent work nevertheless applies such formal methods as computer models of defeasible inference to reasoning and argument (e.g., Walton \& Gordon, 2015).
} 
Blair 2012; van Eemeren et al., 2014: 381-384), where the RSA criteria explain other normative qualities of argument. ${ }^{3}$ Logical validity, for example, is one among other possible standards of inferential sufficiency; probability-raising may serve as a standard of premise relevance; truth may serve as a standard for premise acceptability. Fallacies are often explained in terms of some characteristic failure to meet one or more of the RSA criteria. And, expanding the sufficiency criterion leads to including considerations of dialectical adequacy (e.g., successfully answering pertinent criticisms or objections).

In defeasible arguments, of course, both evaluative factors (premise adequacy and inferential connection) are subject to weakening, strengthening, and defeat, upon introducing new information. Inductive logic is the branch of formal logic dealing with inferences whose validity is sensitive to such dynamics. Yet, having rejected deductive logic in favor of a non-formal approach to argument analysis and appraisal, informal logicians have typically neglected the formal tools provided by inductive logic.

Following Spohn (2012), here we take inductive logic to include all non-deductive logics, including default, non-monotonic, belief-revision, auto-epistemic, agent-based, modal logic, etc. For all these logics concern the support relation holding, or not, between information states A, B, C, D, where A might provide (some) support for D, while $A \& B$ might fail to support $D$, but perhaps support not-D, and A\&B\&C could support D more, or less, than A alone did, etc. Probability theory (see Section 3 ) thus makes for a quantitative variant of inductive logic. ${ }^{4}$

\subsection{The probabilistic approach to argumentation}

Applying probability theory to natural language argument requires extending the notions of evidence and hypothesis to, respectively, reasons and claims, yielding a model of the probative impact of reasons onto claims. ${ }^{5}$ One assumes that an agent

\footnotetext{
${ }^{3}$ Following the introduction of the RSA criteria by Johnson and Blair in 1977 (Johnson \& Blair, 2006: 55), many informalists have adopted, modified, or augmented them (see Johnson \& Blair, 2002: 370). For instance, Govier (2010: 87ff.) calls sufficiency good grounds; Johnson (2000: 189ff.) added premise truth as a fourth criterion, situating this together with adequacy at the "dialectical tier;" Vorobej (2006: 49ff.) replaced acceptability with truth and added compactness as a fourth criterion to stipulate the absence of irrelevant premises.

${ }^{4}$ Spohn's own ranking theory (Spohn, 2012) also qualifies as an inductive logic. Pursuing a Baconian approach to probability, his theory is more general than the Pascalian approach we rely on. (For these terms, see Cohen, 1989.) Ranking theory models the differential retractability of full rather than graded propositions, interpreted as belief-contents. By contrast, we speak of graded commitments to reasons or claims.

${ }^{5}$ The more common interpretation - 'the probability of a hypothesis, $H$, given evidence, $E$ ' - reflects the role of the probability calculus for the empirical sciences when gauging the (dis-)confirmatory effect of evidence on hypotheses, calculation of which relies on Bayes' theorem. Several differences arise in contexts of defeasible inference and argumentation: First, scientific hypotheses typically have a predictive or explanatory relationship to the evidence. Second, the evidence here typically accumulates through independent instances (e.g., observations or test results), making the reliability of evidence expressible as long-run frequencies. Neither feature need hold between a claim and the reasons offered in its support. Finally, as Strevens (2012: 23; notation adapted) writes: "a Bayesian conditionalizes on [some evidence] $E$ - that is, applies Bayes' rule to $E$-just when they 'learn that $E$ has occurred.' In classical Bayesianism, to learn $E$ is to have one's subjective probability for $E$ go to one [i.e., a probability value of 1, denoting
} 


\section{DAVID GODDEN AND FRANK ZENKER}

responds to a reason by updating her prior belief in - or as we prefer to say: commitment to - a claim, in order to form a posterior commitment by conditionalizing on the reason (see Godden, 2010). The support that a reason $R$ offers to a claim $C$ (see Howson $\&$ Urbach, 2006: 92) provides a measure of argument force. It can be expressed as the difference between the prior probability of $C$ independently of the reason, $P(C)$, and the posterior, or final $(f)$, probability of $C$ conditional on the reason, $P_{f}(C)=P(C \mid R)$, i.e., $P_{f}(C)-P(C)$ (see Korb, 2004: 44). Other measures remain possible (see Fitelson, 2001; Pfeifer, 2013: 187ff.). Moreover, the value to which $P_{f}(C)$ is set upon the uptake of $R$ measures argument strength, such that:

Argument strength, then, on this [probabilistic] account is a function of the degree of prior conviction, $P(C)$, and the relationship between the conclusion and the evidence, in particular how much more likely the evidence would be if the conclusion were true, $P(R \mid C)$. (Hahn \& Oaksford, 2007: 707; notation adapted)

To a normative account that views argument primarily as an abstract inferential object, rather than as a process of dialectical exchange, the central research question is: "How much change in existing beliefs should new evidence [in the form of reasons] bring about?" (Corner \& Hahn, 2013: 3586, italics added). ${ }^{6}$ Of course, one cannot properly assess a particular saturation of an argument scheme by considering the scheme's structural properties (i.e., the argument form) alone, but must also engage with its semantic content. The probabilistic [0,1]-interval now provides the nuances to express that speakers and audiences may accept the contents of reasons, claims, and inferential links but to a matter of degree.

Recent work has provided probabilistic analyses of various classical fallacies, including: appeal to popularity (ad populum) (Korb, 2004), arguments against the person (ad hominem) (Korb, 2004), the 'causal' fallacy (post hoc ergo propter hoc) (Korb, 2004), arguments from ignorance (argumentum ad ignorantiam) (Hahn \& Oaksford, 2006a; Hahn, Oaksford, \& Bayindir, 2005; Oaksford \& Hahn, 2004), circular reasoning (petitio principii or begging the question) (Hahn, Oaksford, \& Corner, 2005), slippery slope arguments (Corner, Hahn, \& Oaksford, 2006), and denying the antecedent and affirming the consequent (Korb, 2004, Godden \& Zenker, 2015). (For an overview, see Korb, 2004; Zenker, 2013.) Hahn and Oaksford (2006a; 2007) argue that a probabilistic approach makes significant progress towards a unified epistemic treatment of the fallacies (Ikuenobe, 2004). Corner and Hahn (2013) make the case for the general suitability of a probabilistic approach to argumentative norms. Most recently, Hahn and Hornikx (2016) have analyzed three argument schemes in the sense of Walton, Reed and Macagno (2008): the argument from sign, and two arguments from testimony, namely the argument from expert opinion (appeal to authority or

certainty] as the result of some kind of observation." By contrast, reasons appearing as premises of an argument need not be certain or unretractable (see Section 3.8). Rather defeasible reasoning and argument involves making judgements about the acceptability of one's premises. Sometimes an update will occur when a reasoner comes to find their reasons are more (or less) acceptable than they did previously.

${ }^{6}$ As Hahn \& Oaksford (2006b: 3) acknowledge, rule-based procedural accounts of argumentation are nonetheless required for the stronger argument (as identified) to in fact "carry through" to the discussion outcome. 
argumentum ad verecundiam) and appeal to popular opinion (argumentum ad populum).

The Bayesian approach to argumentation retains the insight that, independently of contextual considerations, defeasible arguments, including virtually all fallacies, vary in strength as a function of their content. Since argument evaluation pertains to whether a particular saturation of an argument form should be (perceived as) stronger or weaker than another saturation of the same form, the normative yardstick of the probabilistic machinery delivers the verdict sought, given assumptions. Moreover, such predictions have been subject to empirical parameter-estimation which demonstrate-pace the caveats of empirical research - that humans are sensitive to experimental manipulations of argument strength, and that their sensitivity can mirror what the probability calculus prescribes (e.g., Harris et al., 2015).

\section{The probabilistic framework}

\subsection{Overview}

Largely following the introduction by Strevens (2012), this section outlines the basics of Pascalian probability (3.2), its interpretation (3.3), addresses dynamic considerations (3.4), introduces Bayes' theorem (3.5), the notion of impact (3.6) and its interpretation (3.7), and finally turns to Jeffrey conditionalization (3.8), which Section 4 applies to a probabilistic analysis of argument cogency.

\subsection{Basics of Pascalian probability}

Probability theory quantitatively represents the chances assigned to events in a space closed under union (disjunction), intersection (conjunction), and complement (negation). The axioms of probability — whether they are Cox's $(1946 ; 1961)$ postulates or Kolmogorov's (1933), used below_also formalize how new information should affect the probability for these events. The rationale for ascribing normative status to probability theory is that "bending" to these axioms guarantees avoiding sure losses in betting games known as Dutch book-scenarios (Hahn, 2014; Hajek, 2008; cf. Douven \& Schupbach, 2015).

On the assumption that the probability of at least one event in an event space occurring is 1 , a probability function $P(\phi)$ assigns to any other event $\phi$ a value from the $[0,1]$ interval. Hence, $0 \leq P(\phi) \leq 1$ where the initial or unconditional probability $P(\phi)$ takes into account all the background information available to an agent at the time. In a finitely additive space such as Kolmogorov presupposed, moreover, it holds for mutually incompatible events, $A$ and $B$, that the probability of their disjunction is the sum of their individual probabilities: ${ }^{7}$

$$
P(A \vee B)=P(A)+P(B)
$$

[Law of addition]

\footnotetext{
${ }^{7}$ For mutually consistent events, the law is: $P(A \vee B)=P(A)+P(B)-P(A \& B)$.
} 
A consequence is that the probability of an event and the probability of its negation sum to one. For example, since $(A \vee \sim A)$ is a logical truth, its probability is 1 . Thus,

$$
P(\sim A)=1-P(A)
$$

[Complement rule]

\subsection{Interpreting probability in contexts of natural language argument}

For this machinery to apply to natural language argument, we rely on these definitions:

$C=$ claim, conclusion, or standpoint

$R=$ reason, or the set of conjoined premises $\left\{R_{1} \& R_{2} \& \ldots \& R_{n}\right\}^{8}$

$P=$ probability (a measure of credence, subjective belief, or committment)

$P(C \mid R)=$ probability of claim given reason

$t=$ an arbitrary threshold value

When interpreted subjectively, probability values represent degrees of belief, i.e., credences, or graded commitments in reasons and claims, rather than objective chances of events. It makes for a regular objection that ordinary reasoners do not experience or treat their own doxastic or dialectic attitudes as allocations of precise numeric values. How, then, shall one understand probability values? Theorists have offered a behavioral or dispositional explanation in terms of an agent's practical judgements and activities. Ramsey (1931), for instance, explained partial commitment to a claim in terms of the odds at which one would accept a bet that the claim is true.

Argumentation theorists can perhaps best interpret probability values as a measure of retractability. Reasons or claims to which one assigns the extremal values 1 or 0 are unrevisably true or false - one's commitment here is unretractable. However, even a practical or moral certainty in a claim (that one would unconditionally act on) need not entail that one never retracts it; rather, one does not envision circumstances under which one would. Therefore, commitments can well approach 1, but still remain open to revision. Similarly, only claims categorically inconsistent with known evidence receive a probability of 0 - commitment being again unretractable. Finally, commitment for exceptionally improbable claims (e.g., skeptical scenarios) can approach 0 , thus failing to merit consideration in practical circumstances, but remain open to revision.

Within the revisability range $0<P(\phi)<1$, then, probability values express retractability judgements for reasons or claims, and their contradictories or contraries. This allocation needn't be numerically precise, only as precise as the situation calls for. For some purposes imprecise probabilities (Bradley, 2015) can be used, while for others a rough-hewn allocation in qualitative terms (e.g., highly unlikely, more likely than not, doubtful) may well do, particularly when natural language qualifiers are taken to express modifications to an unqualified commitment. In the following, however, we assume that a precise probability value is available.

The above constraints and their consequences guarantee a synchronically consistent distribution of probability values across one's commitments. We now turn to diachronic constraints, i.e., update-rules.

\footnotetext{
${ }^{8}$ We use ' $R$ ' and 'the / a reason' to indicate the conjunction of all the articulated premises of an argument or piece of reasoning.
} 


\subsection{Conditionalization}

Changes in subjective probability can occur inferentially or non-inferentially. Noninferentially, for instance, one may update one's commitment in a claim as the result of an observation (verdical or not). Besides the rules in Section 3.2, however, the probability calculus does not provide constraints for a non-inferential update of probabilities. It is rather assumed that agents who learn new information set a probability value for it, typically updating to a final probability of nearly 1 .

When representing an inferential update via conditionalization on new information (gained inferentially or non-inferentially) as: ${ }^{9}$

$$
P(A \mid B)=\frac{P(A \& B)}{P(B)} \quad[\text { Definition of conditional probability }]
$$

one can model the effects of reason-giving typical in contexts of natural language argumentation as a commitment-update on the exclusive basis of premissory information. Expressing the support offered by a reason $R$ to a claim $C$ as the conditional probability of $C$ given $R$, i.e., $P(C \mid R)$, the final, or posterior, probability of $C, P_{f}(C)$, is then given as:

$$
P_{f}(C)=P(C \mid R)
$$

[Bayes'Rule]

which one may compare to the initial, or prior, probability of $C$ irrespective of $R, P(C)$.

Whether and how accepting $R$ at some positive credence, $P_{f}(R)>0$, should affect one's commitment to $C, P(C)$, now depends on factors discussed below.

\subsection{Bayes' Theorem}

Equation (3) defined the posterior probability of a claim $C$ given a reason $R$ as the probability of the claim and the reason, divided by the probability of the reason irrespective of the claim - here repeated, with adapted notation, as (5):

$$
P(C \mid R)=\frac{P(C \& R)}{P(R)}
$$

Now the probability of two events, $A$ and $B$, both occurring is the probability that one of them, $A$, occurs if the other, $B$, does, times the probability that $B$ occurs, as in (6): ${ }^{10}$

\footnotetext{
${ }^{9}$ (3) says that the probability of event $A$ occurring, given some other event $B$ does, equals the probability of both events occurring, divided by the probability that event $B$ occurs anyways. Since the probability of any two events occurring is never greater than the probability of either event occurring individually, $P(A \& B) \leq P(B)$ holds. This guarantees a probability value in the range $0 \leq P(A \mid B) \leq 1$, so long as $P(B)<0$. If $P(B)=0$ then $P(A \mid B)$ is undefined.

${ }^{10}$ In cases where $A$ and $B$ are independent, such that there is no systematic positive or negative correlation between them, it holds that: $P(A \mid B)=P(A)$ and $P(B \mid A)=P(B)$.
} 


$$
P(A \& B)=P(A \mid B) \times P(B)
$$

Since conjunction is commutative (i.e., 'A\&B' and ' $\mathrm{B} \& \mathrm{~A}$ ' state logically equivalent contents), we may now derive Bayes' Theorem (BT) (Bayes 1763) by substituting, notation adapted, the right hand side of (6) into the numerator of (5):

$$
P(C \mid R)=\frac{P(R \mid C) \times P(C)}{P(R)}
$$

[Bayes' Theorem (BT)]

(8) simply separates the prior probability of the claim, $P(C)$, from the numerator in $(7):^{11}$

$$
P(C \mid R)=P(C) \times \frac{P(R \mid C)}{P(R)}
$$

This provides a perhaps more intuitive way of understanding how, in generating the conditional probability, the reason has impact upon the prior probability of the claim, to which we now turn.

\subsection{The impact term in Bayes' Theorem}

The factor by which the prior probability of a claim $C$ is to be multiplied in order to yield the posterior probability of $C$ conditional on the reason $R$, we call the impact of the reason, $i:^{12}$

$$
i=\frac{P(R \mid C)}{P(R)}
$$

[Impact term]

(9) states the impact term as the ratio of how probable the reason is given the claim, to how probable the reason is irrespective of the claim. This "reason ratio" expresses a conditional expectation of the reason, namely an expectation of the reason if the claim holds as against a prior expectation on the reason regardless. (We return to this ratio in Section 4.) Thus, the posterior probability of a claim is the probability of the claim conditional on the reason, which is its prior probability times the impact of the reason:

$$
P_{f}(C)=P(C \mid R)=P(C) \times i
$$

[BT with impact term]

Applying the law of total probability: ${ }^{13}$

\footnotetext{
${ }^{11}$ (8) states that the posterior probability of $C$ given $R$ is the prior probability of $C$ times the likelihood of the reason given the claim, $P(R \mid C)$, over the probability of the reason, $P(R)$. (The notion of likelihood is introduced in Section 3.7.)

${ }^{12}$ Joyce (2009: 5) notes that Carnap (1962: 466) identified $i$ as the relevance quotient, or the probability ratio; Strevens (2012: 30) calls $i$ the Bayes multiplier.

${ }^{13}$ (11) states the chance of event $A$ occurring as the chance that $A$ occurs given another event $B$ does, times the chance that $B$ occurs, plus the chance that $A$ occurs given $B$ fails to occur, times the chance that
} 


$$
P(A)=P(A \mid B) \times P(B)+P(A \mid \sim B) \times P(\sim B) \quad[\text { Law of Total Probability }]
$$

to the denominator of $i$, in (9), yields, notation adapted, the "long version" of BT: ${ }^{14}$

$$
P(C \mid R)=P(C) \times \frac{P(R \mid C)}{P(R \mid C) \times P(C)+P(R \mid \sim C) \times P(\sim C)}
$$

which allows (see Howson \& Urbach, 2006: 97; Korb, 2004: 44) expressing the impact term $i$ as:

$$
i=\frac{P(R \mid C)}{P(R \mid C) \times P(C)+P(R \mid \sim C) \times P(\sim C)}
$$

So far, we have mainly sought to make textbook-knowledge more palatable to the tastes of informal logicians. In (13) the priors $P(C)$ and $P(\sim C)$ report prior commitments to the truth or falsity of $C$, given background information, where $P(\sim C)=1-P(C)$. However, suitably interpreting the remaining two terms of (13), which both express likelihoods, is more challenging.

\subsection{Interpreting the impact term}

Likelihoods express prior judgements about the probative value of reasons, subject to the constraints: $P(R \mid C)=1-P(\sim R \mid C)$, and $P(R \mid \sim C)=1-P(\sim R \mid \sim C) .{ }^{15}$ The first likelihood term, $P(R \mid C)$, expresses the sensitivity of the reason to the claim. When evaluating the reliability of an empirical test, for instance, and given that 'hypothesis $(\mathrm{H})$ ' replaces 'claim' and 'evidence (E)' replaces 'reason', this same term reports the true positive rate (i.e., the ratio of correct positive test-results over all test-results). The second likelihood term, $P(R \mid \sim C)$, expresses the complement of the specificity of the reason to the claim. ${ }^{16}$ Also called the false positive rate of a test, it reports the ratio of incorrect positive test-results over all test-results. Considered together, both likelihood terms express how well $R$ correlates with $C$. For example, if $C$ entails $R$, then $P(R \mid C)=1$; if no correlation obtains, then $P(R \mid C)=P(R \mid \sim C)=P(R)$ (see Section 4.2).

$B$ fails to occur. The law presupposes conditionalization on exhaustive alternatives, which any claim $B$ and its negation $\sim B$ of course are.

${ }^{14}$ (12) says that the posterior probability of a claim $C$ given a reason $R$ is the prior probability of $C$ times the likelihood of $R$ given $C$, divided by the sum of the likelihood of $R$ given $C$ times the probability of $C$, and the likelihood of $R$ given not $C$ times the probability of not $C$.

15 While equation (2) was a consequence of the logical truth $(A \vee \sim A)$, these two constraints are consequences of the metaphysical truth that, given event $B$ occurs, any other event $A$ will either occur, or not.

${ }^{16}$ Notice that, since $P(R \mid \sim C)=1-P(\sim R \mid \sim C)$, rather than using 'the logical complement of specificity' in order to refer to $P(R \mid \sim C)$, we use the term 'specificity' alone. 
Sensitivity and specificity are readily meaningful for long-run frequencies of event tokens. However, as Hahn and Oaksford (2007: 714; italics added) rightly note,

much of the evidence adduced in an everyday argument will relate to singular events. For example, an argument over who killed Kennedy will have to appeal to many events that can also have happened only once, for example, what is the probability that Oswald was hired by the Mafia? Consequently, to provide a general probabilistic account of argument strength requires assigning single event probabilities, which only makes sense from a Bayesian subjective perspective. Single event probabilities cannot, by definition, be affected by the amount of evidence in the sense of a simple enumeration of positive instances.

Of course, both likelihood terms must remain meaningful if reasons shall provide support for claims irrespective of frequency considerations.

Rather than opt for frequencies, the following interpretation is more useful in contexts of natural language argumentation: reason $R$ is sensitive to claim $C$ to the extent that $R$ supports $C$ more than $R$ supports any other claim, $C^{*}$, that itself entails $\sim C$, i.e., $P(C \mid R)>0.5>P(\sim C \mid R)$. And $R$ is specific to $C$ to the extent that $R$ rather than any other reason, $R^{*}$, itself entailing $\sim R$, supports $C$, i.e., $P(C \mid \sim R)<0.5<P(\sim C \mid \sim R)$. Drawing considerations of sensitivity and specificity together, the support that $R$ generates for $C$ thus depends on the extent to which the $C$-supporting-reason $R$ fails to support $\sim C$, on one hand, and on the extent to which argumentative support for $C$ cannot be generated by reasons besides $R$, on the other. In the extremal cases $P(C \mid R)=1$ and $P(C \mid R)=0$, support is thus strongest where $R$ is an exclusive and decisive supporting reason-for- $C$, and weakest where $R$ is a common and indecisive supporting reason-for-C.

This should become clearer below. Presently, consider as an example of an exclusive and decisive supporting reason (outside the argumentative domain), a modern litmus test in the form of a universal $\mathrm{pH}$-indicator (hydrogen ion), where the red coloring of the indicator paper is a causal effect of a solution's hydrogen ion concentration. Assume, unrealistically, that the test is perfectly sensitive, i.e., $P(R \mid C)=1$, since any $\mathrm{pH}$-level below 3 always causes the indicator paper to turn red, and also perfectly specific, i.e., $\mathrm{P}(C \mid \sim R)=0$, since other $\mathrm{pH}$-levels always color the paper non-red. Equally unrealistically, assume that no other test for the same purpose is available. Now the paper's not turning red decisively indicates that the solution is not strongly acidic, so $P(\sim R \mid \sim C)=1-P(R \mid \sim C)=1$ - and exclusively, too, since (by assumption) no other test can. In this case, from equation 13, $i=1 / P(C)$; and thus BT (equation 12) reports $P(C \mid R)=1$.

As an example of a common indecisive supporting reason, consider using the results of a fair coin toss as as a reason-say, taking a coin's landing heads as a reason for the claim that the time at which the coin lands (to the 100th of a second), is an even number. Assume, this time realistically, that no correlation holds, so that the "test" is perfectly insensitive, the coin landing heads if the time is even thus being as probable as it landing tails. Hence, $P(R \mid C)=1-P(\sim R \mid C)=0.5$. Likewise, the test is perfectly unspecific: when the number is even, then the chances are the same that the coin lands tails as that it lands heads. Hence, $\mathrm{P}(\sim R \mid \sim C)=\mathrm{P}(R \mid \sim C)=0.5$. In this case, from (13), $i=1$, and thus BT (equation 12) reports $P(C \mid R)=P(C)$. 


\subsection{Jeffrey Conditionalization}

So far, conditional update has been explained on the assumption that the reason is accepted as unretractably true, i.e., $P_{f}(R)=1$. BT operationalizes conditionalization on the assumption that "one acquired new evidence which can be represented as becoming certain of an evidentiary statement $E$ [read: reason $R$ ]" (Talbot, 2011: 3). Ordinary reasoning and argument can satisfy this assumption (e.g., when learning new information through perceptual experience, from a testimonial report, test result, or instrument reading). In other cases, however, arguers accept reasons - their own or others' - not without qualification, but place some graded measure of commitment in them. Hence, commitment-update should occur proportionally to partial commitment.

Jeffrey Conditionalization (JC) (Jeffrey, 1983) allows conditionalizing on a partial commitment in a reason. When updating from $P(R)$ to $P_{f}(R)$, JC prescribes that one conditionalize as follows: ${ }^{17}$

$$
P_{f}(C)=P(C \mid R) \times P_{f}(R)+P(C \mid \sim R) \times P_{f}(\sim R) \quad[J C]
$$

Notice that, if $P_{f}(R)=1$, then $P_{f}(\sim R)=0$, so that (14) reduces to its left summand, yielding Bayes' Rule (see equation 4). Now substituting BT (equation 8 ) for the conditionalized probabilities $P(C \mid R)$ and $P(C \mid \sim R)$ in JC yields:

$$
P_{f}(C)=\frac{P(R \mid C)}{P(R)} \times P(C) \times P_{f}(R)+\frac{P(\sim R \mid C)}{P(\sim R)} \times P(C) \times P_{f}(\sim R)
$$

Further, isolating $P(\mathrm{C})$ from each summand in (15) yields:

$$
P_{f}(C)=P(C) \times\left[\frac{P(R \mid C)}{P(R)} \times P_{f}(R)+\frac{P(\sim R \mid C)}{P(\sim R)} \times P_{f}(\sim R)\right]
$$

which is equivalent to: ${ }^{18}$

$$
P_{f}(C)=P(C) \times\left[P(R \mid C) \times \frac{P_{f}(R)}{P(R)}+P(\sim R \mid C) \frac{P_{f}(\sim R)}{P(\sim R)}\right]
$$

Hence, from (16), when updating on a partial commitment, the impact term $i$ becomes:

\footnotetext{
${ }^{17}$ (14) says that the final commitment in $C$, updated on a partial commitment in $R$, is the commitment in $C$ given $R$, times the final commitment in $R$, plus the commitment in $C$ conditional on $\sim R$, times the final commitment in $\sim R$. When conditionalizing on a partial commitment in order to update, JC thus recognizes that a partial commitment in $R$ is a partial commitment in $\sim R$, which together sum to one.

${ }^{18}$ (17) states that one's final commitment in $C$ conditionalized on one's final commitment in $R$, where $0<P_{f}(R)<1$, is one's initial commitment in $C$, times the sum of the likelihoods that $R$ given $C$, multiplied by the ratio of one's final commitment to the reason over one's initial commitment to it, and the likelihood of $\sim R$ given $C$, multiplied by the ratio of one's final commitment that $\sim R$ over one's initial commitment to it.
} 


$$
i=\left[\frac{P(R \mid C)}{P(R)} \times P_{f}(R)+\frac{P(\sim R \mid C)}{P(\sim R)} \times P_{f}(\sim R)\right]
$$

or, rewritten, from (17):

$$
i=\left[P(R \mid C) \times \frac{P_{f}(R)}{P(R)}+P(\sim R \mid C) \frac{P_{f}(\sim R)}{P(\sim R)}\right]
$$

Notice, most obviously in (18), that the JC-impact term is equivalent to the impact term in BT if $P_{f}(R)=1$, since the right hand summand of (18) then goes to zero.

This in place, we now apply the forgoing to give an analysis of cogency in the evaluation of natural language argumentation.

\section{Cogency}

\subsection{The RSA criteria}

Informal logicians analyze argument cogency as three individually necessary and jointly sufficient conditions: acceptability, relevance, and inferential sufficiency. This section provides a probabilistic view upon each condition, starting with relevance (4.2), then treats sufficiency (4.3), comparing it with informal accounts (4.4), and finally turns to acceptability (4.5).

\subsection{Relevance}

Relevance standardly counts as an independent criterion of cogency. ${ }^{19}$ The basic idea is that a relevant reason provides some support for a claim. As Govier (2010: 148; notation adapted) puts it "[a] statement $R$ is positively relevant to another statement $C$, if and only if the truth of $R$ counts in favor of the truth of $C$." Hence, $C$ given $R$ must be more probable than otherwise, i.e., $P(C \mid R)>P(C)$, yielding a spectrum of relevance:

$$
P(C \mid R)\left\{\begin{array}{l}
>P(C) \\
=P(C) \\
<P(C)
\end{array}\right.
$$

[ $\mathrm{R}$ is positively relevant to $\mathrm{C}]$ [ $\mathrm{R}$ is irrelevant to $\mathrm{C}$ ] [ $\mathrm{R}$ is negatively relevant to $\mathrm{C}$ ]

An equivalent measure, as we saw in equation 10, is:

$$
P(C \mid R)=P(C) \times i
$$

\footnotetext{
19 Granted that sufficiency presupposes relevance, reasons by contrast can be relevant without being sufficient. Indeed, distinct relevance-based failures of arguments are identified by the fallacies of relevance (Johnson \& Blair, 2002: 370). Johnson (2000: 200) claims that the notion of relevance it itself "basic" and "a ground-floor notion that a reasoner must grasp." See Powers (1995) for the view that all fallacies allegedly are nothing but relevance problems. Zenker (2016) provides an overview of what currently does (not) count as a fallacy.
} 
which expresses relevance in terms of the impact term $i$, where (from equation 18) we know that $i=\left[\frac{P(R \mid C)}{P(R)} \times P_{f}(R)+\frac{P(\sim R \mid C)}{P(\sim R)} \times P_{f}(\sim R)\right]$, such that:

$$
i\left\{\begin{array}{l}
>1 \\
=1 \\
<1
\end{array}\right.
$$

[ $\mathrm{R}$ is positively relevant to $\mathrm{C}$ ] [ $\mathrm{R}$ is irrelevant to $\mathrm{C}]$ [ $\mathrm{R}$ is negatively relevant to $\mathrm{C}$ ]

Defining relevance via the impact term makes relevance a function of the priors in the expectation of the reason (see Section 3.6), which one may calculate prior to update. This operationalizes an intuitive notion of relevance: if $R$ is just as probable whether or not $C$ - for which $R$ is putatively a reason - then $R$ is neither positively nor negatively correlated to $\mathrm{C}$. Hence, $R$ is irrelevant to $C$.

This explains why random information, where $P_{f}(R)=1$, (e.g., the result of a fair coin toss) cannot supply a relevant reason (see the example in Section 3.7). In such cases $P(R \mid C)=P(R)$; hence $i=1 .^{20}$ Likewise for logically true reasons (e.g., tautologies): if $P(R)=1$, then by preservation of certainties (Joyce, 2009: 2) we have it that $P(R \mid C)=1$. We can similarly explain the relevance problem of ex falso quod libet ("from a contradiction anything follows"): while one cannot conditionalize on a reason of probability zero, one can recognize that a contradiction is equally improbable in cases where the claim at issue obtains as otherwise. In all these cases, the putative reason does not correlate with the claim. Hence, if $i=1$, then $R$ has no impact on $C$, i.e.,

$$
(i=1) \rightarrow[P(C \mid R)=P(C)]
$$

\subsection{Inferential sufficiency}

Informal logicians contend that a reason provides sufficient support to a claim $C$ if, and only if, the probability of the claim meets or exceeds a threshold, $t_{S}$, when one accepts $C$ because of $R$. Initially, we can define sufficiency as: ${ }^{21}$

$$
P_{f}(C)=P(C \mid R) \geq t_{s}>P(C)
$$

[inferential sufficiency]

The latter condition, $t_{S}>P(C)$, is significant since sufficiency measures whether the claim is acceptable on the basis of the reason provided in the argument, rather than otherwise, e.g., when the claim is already acceptable independently.

Though the exact value of $t_{S}$ ever depends on context, it nevertheless generally holds that $0<t_{S}<1$, for a claim certainly known to be false, i.e., $P_{f}(C)=0$, ought not to be accepted, while a claim certainly known to be true, i.e., $P_{f}(C)=1$, ought not to be rejected. Next, if a reason deductively entails a claim, i.e., $\mathrm{R} \mid-\mathrm{C}$, and if $R$ is known to be

\footnotetext{
${ }^{20}$ In cases of irrelevance, both the impact term and the likelihood ratio, $P(R \mid C) / P(R \mid \sim C)$ equals 1 (see Korb, 2004: 44; Hahn \& Hornikx, 2016: 1838). Where $i=1$, it follows that $P(R \mid C)=P(R)$; so by the law of total probability (equation 11): $P(R \mid C)=P(R \mid \sim C$ ).

${ }^{21}$ (24) says that the final probability of $C$ is the posterior probability of $C$ given $R$, which meets or exceeds the threshold, $t_{s}$, and exceeds the prior probability of $C$.
} 
true, i.e., $P_{f}(R)=1$, then $P_{f}(C)=1$ (see Joyce, 2009: 2). ${ }^{22}$ If $R$ entails $C$, yet the commitment to $R$ is uncertain, i.e., $0<P_{f}(R)<1$, one might think that $P_{f}(C)=P_{f}(R)$. But as JC (see equation 14) shows, this need not hold, for $P_{f}(C)$ depends on both $P_{f}(R)$ as well as on the extent to which $C$ is supported or refuted by $\sim R$, i.e., $P(C \mid \sim R)$ and $P_{f}(\sim R)$.

One might further expect that sufficient reasons should normally make claims more probable than not, such that $1>P(C) \geq t_{S}>0.5$, that is, sufficient reasons should make claims acceptable on balance of probabilities. On a balance of probability threshold, $t_{S}=0.5+\varepsilon$, where $\varepsilon$ is some arbitrarily small quantity. But it is easy to conceive of situations where $t_{S}$ exceeds $0.5+\varepsilon$ (e.g., 'is highly probable', or 'to a moral certainty', or 'beyond reasonable doubt', etc.), or where $t_{S}$ falls below $0.5+\varepsilon$ (e.g., when a precautionary principle prompts considering the significant disutility of an otherwise improbable claim).

In (24) inferential sufficiency is defined as a minimum acceptability threshold, $t_{S}$, for $P_{f}(C)=P(C \mid R)$. BT (equation 10) tells us that $P(C \mid R)$ can be equivalently expressed as $P(C) \times i$, such that, in cases of inferential sufficiency:

$$
P(C) \times i \geq t_{s}>P(C)
$$

Since (25) stipulates that:

$$
P(C) \times i>P(C)
$$

sufficiency entails that:

$$
i>1
$$

Hence, sufficiency entails, and so is a logically stronger condition than, positive relevance.

Furthermore, like relevance, one can define inferential sufficiency in terms of impact, $i$. Dividing each term in $(25)$ by $P(C)$ yields:

(28) $i \geq \frac{t_{s}}{P(C)}>1$

[Sufficiency defined in terms of impact]

and so gives the following spectrum of inferential sufficiency:

$$
i\left\{\begin{array}{lr}
>\frac{t_{s}}{P(C)} & \text { [R is a supererogatory reason for } \left.C \text { relative to } t_{s}\right] \\
=\frac{t_{s}}{P(C)} & {\left[\mathrm{R} \text { is a sufficient reason for } \mathrm{C} \text { relative to } t_{s}\right]} \\
<\frac{t_{s}}{P(C)} & {\left[\mathrm{R} \text { is an insufficient reason for } \mathrm{C} \text { relative to } t_{s}\right]}
\end{array}\right.
$$

${ }^{22}$ After all, if $R$ entails $C$, then $P(C \& R)=P(R)$, and given the definition of conditional probability (equation 3), we have it that $P(C \mid R)=1$. 
As a special case of an insufficient reason, consider that $R$ is a necessary reason-for-C whenever some reason-for- $\mathrm{C}, Q$, in fact provides no support to $C-$ so $P_{f}(C \mid Q)=P(C)$ unless $P(R)=1$, that is, $1=P(R) \geq P_{f}(C \mid Q \& R) \geq t_{s}>P_{f}(C \mid R) \geq P_{f}(C \mid Q)=P(C) \geq 0$. Where $R$ is a sine qua non reason-for-C, moreover, we also have it that $P_{f}(C \mid \sim R)=0$. (Compare Spohn, 2012: sect. 6.1, 104 ff.).

\subsection{A probabilistic corrective on threshold applications of sufficiency}

In (24) $t_{S}$ is expressed as a minimum threshold value for the acceptability of a claim. Since informal logicians treat inferential sufficiency as a necessary condition for cogency, arguments citing insufficient reasons for their conclusions are non-cogent, and ought not to be accepted. Yet, what 'accepting an argument' means or amounts to is not entirely clear.

On one reading, 'accept' refers to the correct prescription that one ought not to endorse an insufficiently supported claim. By contrast, those who understand offering arguments as the issuing of "invitations to inference" (Pinto, 2001: 36-37) can interpret the sufficiency criterion as prohibiting any inferential use of reasons failing the threshold. On this other reading, one should not update inferentially unless the sufficiency condition (e.g., as given in equation 28) is satisfied.

Call this restriction a threshold application of inferential sufficiency. Indeed, if one should only act inferentially on reasons if they satisfy the sufficiency condition (and, $a$ fortiori, the relevance condition), then a sufficiency condition acts as an inference gate: what meets or exceeds the threshold should occasion inferences, but not the rest. So inference gates exclude information. Yet, this could be a mistake.

To see this, consider-as Example 1-a situation where one is successively presented with several independent and individually insufficient reasons for a claim. Assume a balance-of-probability sufficiency threshold of $t_{S}=0.5+\varepsilon$ (see Section 4.3). Suppose that initial commitment to the claim is quite low, $P(C)=0.17$, as are commitments to a set $\boldsymbol{R}=\left\{R_{1}, R_{2}, R_{3}, R_{4}\right\}$ of four logically independent reasons, such that $P\left(R_{1}\right)=P\left(R_{2}\right)=P\left(R_{3}\right)=P\left(R_{4}\right)$. (Recall from Section 3.3 that ' $R$ ' ('the reason') indicates the set of conjoined premises of some argument.) Further, suppose each reason in $\boldsymbol{R}$ is equally weakly indicative of the claim, say $P\left(R_{n} \mid C\right)=0.25$, and that each reason's false positive rate is quite low, say $P\left(R_{n} \mid \sim C\right)=0.15$. The law of total probability (equation 11) now yields the initial probability of any reason in $\boldsymbol{R}$ as $P\left(R_{n}\right)=0.167 .^{23}$ Upon learning that any one of the reasons $R_{1}$ to $R_{4}$ (say $R_{1}$ ) obtains, according to BT (equation 8) our commitment in $C$ should change-from $P(C)=0.17$ to $P_{1}(C)=0.2545$, where ' $P_{n}(\phi)$ ' denotes the $n^{\text {th }}$ revision of $P(\phi)$. Yet, since $P_{1}(C)<0.5+\varepsilon$, the updated commitment still fails to satisfy the sufficiency threshold. So, if equation 28 states an inference gate, then although each reason by itself $i$ s relevant (since $i_{R 1}=1.497>1$ ), we should nevertheless not act inferentially because by itself any reason $R_{n}$ in $\boldsymbol{R}$ fails to satisfy the sufficiency condition, since $i<t_{S} / P(C)=2.942$.

According to BT, however, updating on the four reasons in succession results in a final credence of 0.6093 , which is above the threshold. To see this, following an initial update on $R_{1}$, the priors on each of the remaining reasons, $R_{2}$ to $R_{4}$, can be

\footnotetext{
${ }^{23}$ For all calculations in Example 1, see the Appendix.
} 
recalculated - by applying the law of total probability on the updated value of $P_{1}(C)$ to yield a new prior $P_{1}\left(R_{2}\right)=0.1755$. A second update, on $R_{2}$, similarly fails to satisfy the sufficiency condition, since BT here yields $P_{2}(C)=0.3625$, where $i=1.425$ and $t_{S} / P_{1}(C)=1.965$. Since it remains the case that $i<t_{S} / P_{1}(C)$, the successive reasons $R_{1}$ and $R_{2}$ combined thus still insufficiently support the claim. Hence, the successive arguments ' $R_{1}$, ergo $C$ ' and ' $\mathrm{R}_{2}$, ergo $\mathrm{C}$ ' are non-cogent; a threshold application of sufficiency would prohibit inferential action. Similarly with a third update, where the values are: $P_{2}\left(R_{3}\right)=0.1875 ; P_{3}(C)=0.4833 ; i=1.333 ; t_{S} / P_{2}(C)=1.379$. Yet, by the fourth update the sufficiency condition $i$ met, for the values are: $P_{3}\left(R_{4}\right)=0.1983 ; P_{4}(C)=0.6093 ; i=1.26$; and $t_{S} / P_{3}(C)=1.035$. Hence, $P_{f}(C) \geq t_{S}$ (where $t_{S}=0.5+\varepsilon$ ), and $i \geq t_{S} / P_{3}(C)$. Notice that, on a threshold application of sufficiency only the fourth update is permitted - and only when the first three have already (but impermissibly) occurred. Yet had one withheld from making initial or intermediate inferential updates - on the ground that each individual reason fails the sufficiency condition-one would have failed to recognize that individually weak reasons can successively accumulate probative force, and thereby achieve sufficiency. ${ }^{24}$ Applying a sufficiency threshold as a necessary precondition of inferential update can therefore mistakenly prevent the update of one's commitments even in cases where, following updates on individually insufficient reasons, a final commitment in the claim satisfies the sufficiency condition.

\footnotetext{
${ }^{24}$ As an anonymous reviewer has rightly pointed out, an informal logician might respond that a set of individually weakly supporting reasons-for-C can, when taken together, provide sufficient convergent support-i.e., individually insufficient but jointly sufficient reasons - to the claim. This might be thought to provide an answer to the challenge to a threshold approach to sufficiency posed by Example 1 .

Yet, on standard accounts, this requires (somehow) taking the independent reasons together all at once, rather than separately in succession. Example 1 shows that, on a probabilistic understanding of inferential sufficiency, this combining of reasons is not required. Rather, the probabilistic calculus offers a formal understanding of how sufficient support can incrementally accrue via a succession of individually insufficient arguments, and so obviously contradicts the "weakest link-principle," also known as Theophrastus' rule (see Hahn \& Oaksford, 2006: 15).

Moreover, since we operationalize inferential sufficiency as an acceptability threshold on the probability of the claim conditional on a reason, $P(C \mid R)$, further issues arise with a similar threshold approach to acceptability that result from interpreting a threshold application of inferential sufficiency as an inference gate (see Examples 2 and 3; Section 4.5). For instance, suppose that the claim in Example 1, now abbreviated as $C_{1}$, does itself provide a reason for another claim, $C_{2}$. As Example 3 will show, even a small sub-threshold change in the acceptability of $C_{1}$ could push $C_{2}$ above a sufficiency threshold when $C_{2}$ is subsequently conditionalized on $C_{1}$. So rather than immediately update on each individually insufficient reason the moment the reason "comes in," to instead collect several weakly supporting reasons-for- $C_{1}$-as if holding these in memory until they (somehow) jointly meet a sufficiency threshold - could preclude a commitment-update in $C_{2}$ under some conditions, although a sufficiency threshold would have been met if the acceptability of $C_{1}$ had been updated earlier.

Future work should provide a probabilistic analysis of the linked vs. convergent support-distinction in informal logic, which we cannot provide here. One possible result pertains to the identity conditions of reasons and premises. For some natural language material may well be discernable as a distinct premise but not count as a distinct reason, namely if offering (or receiving) that premise to support the claim $C$ would, by itself, fail to render $P(C \mid R)>P(C)$. The inferential effect of $R$ on $P(C)$, or the lack thereof, could thus become a criterion for a premise to in fact act as a reason. Hence, the identity conditions of reasons and premises in the probabilistic and the informal logic approach might be the same, while their functional properties could diverge.
} 


\subsection{Premise acceptability}

Premise acceptability appears to be the most straightforward of the cogency conditions to define probabilistically. The basic idea, again, is that acceptable reasons meet or exceed a threshold, $t_{a}$, such that:

$$
P_{f}(R) \geq t_{a}
$$

where $t_{a}$ may, though it need not, also serve as the acceptability threshold for a proper subset of the set of conjoined premises $R .^{25}$ Similar to threshold applications of sufficiency, and facing similar problems, call this a threshold application of acceptability. As with claims, reasons certainly known to be false, i.e., $P_{f}(R)=0$, ought not to be accepted, and reasons certainly known to be true, i.e., $P_{f}(R)=1$, ought to be rejected.

When the acceptability of a reason falls between 0 and 1, one should of course consider all the evidence at hand. Moreover, one should update by conditionalizing on a reason so long as this has positive or negative bearing on a claim. But, again, if one should only act inferentially on reasons that meet a standard of acceptability, then this standard, too, acts as a commitment gate, for it excludes information falling below the threshold. For example, describing the proper application of the cogency criteria, Johnson (2000: 343; emphasis added) writes:

Suppose, for example, that [premise] P1 is irrelevant (or untrue, or unacceptable) - then P1 won't be allowed in the premise-set that I check for sufficiency. ... [B] ecause sufficiency is a global requirement, applying to all the premises taken together, it should not be applied until the premise-set is stabilized; that is, the set will have been inspected already for acceptability, truth, and relevance.

This, too, can be a mistake. To see this, recall JC (equation 17; see Section 3.8):

$$
P_{f}(C)=P(C) \times\left[P(R \mid C) \times \frac{P_{f}(R)}{P(R)}+P(\sim R \mid C) \frac{P_{f}(\sim R)}{P(\sim R)}\right]
$$

When commitments change, but one nevertheless withholds full commitment to some reason, then-since a partial commitment to $R$ is a partial commitment in $\sim R-\mathrm{JC}$ prescribes that one conditionalize on both $R$ and $\sim R$ accordingly. But this does not readily fit with a threshold application of acceptability.

For instance, consider - as Example 2 - an acceptability threshold of $t_{a} \geq 0.85$, and set prior commitments to: $P(R)=1-P(\sim R)=0.2 ; \quad P(C)=1-P(\sim C)=0.3$; $\quad$ and $P(R \mid C)=1-P(\sim R \mid C)=0.8$. Since no prior (including priors on the complements) meets the acceptability threshold, none of these elements count as accepted. Now suppose new

\footnotetext{
${ }^{25}$ The acceptability of a set of reasons is some function of the acceptability of constituent reasons. Those considering the acceptability of individual and conjoint reasons may find that different acceptability standards are in effect (e.g., a higher threshold for the deliverances of reason and sensation than for memory or testimony).
} 
information prompts a revision to $P_{1}(R)=1-P_{1}(\sim R)=0.5$, where, as before, ' $P_{n}(\phi)$ ' denotes the $n^{\text {th }}$ revision of $P(\phi)$. A threshold application still instructs withholding commitment from both $R$ and $\sim R$, and therefore to not act inferentially on either. According to $\mathrm{JC}$, however, commitment in the claim should change, from $P(C)=0.3$ to $P_{1}(C)=0.675$, rendering one's commitment about twice as strong, although still below the acceptability threshold. Now iterate, by updating to $P_{2}(R)=1-P_{2}(\sim R)=0.8$, and the threshold application still requires withholding commitment, so one is not to act inferentially on either $R$ or $\sim R$. But JC now reports $P_{2}(C)=0.867$, which exceeds the acceptability threshold.

Finally consider-as Example 3-that the same effects of relevance and sufficiency can be achieved with only a single, small sub-threshold change in one's commitment to a reason, so long as the reason's link to a claim is strong enough (because the reason is highly sensitive, or its negation highly selective to the claim). In case commitment in a reason changes from $P(R)=0.2$ to $P_{f}(R)=0.4$, where $R$ is highly sensitive to $C$, i.e., $P(R \mid C)=0.8$, and where $\sim R$ is quite selective for $\sim C$, i.e., $P(\sim R \mid \sim C)=0.6$, then although a balance of considerations acceptability threshold on $R$ having not been met after update, $R$ is nevertheless relevant since $i=1.9$ (by equation 19). Further, assuming a sufficiency threshold, $t_{s}=0.9$, and an indifferent (or uninformative) prior on $C$, i.e., $P(C)=0.5$, we have it that $t_{S} / P(C)=1.8$. Since $i \geq t_{S} / P(C)$ (equation 29), $R$ meets the sufficiency criterion, and JC yields a final probability $P_{f}(C)=0.95 \geq t_{S}$.

Thus, applying acceptability thresholds interpreted as necessary preconditions for inferential update would prevent an update of commitments, even though the final commitment in the claim permits acceptance.

\section{Discussion}

\subsection{The RSA conditions}

On the above analysis, relevance, sufficiency and acceptability are neither conceptually primitive nor conceptually independent. Rather, they each depend on a common set of factors. Analyzed in terms of impact (equations 9 and 18), relevance (equation 22) is a function of the change in the acceptability of a reason, together with its sensitivity to, and its selectivity for, the claim. Sufficiency (equation 29) is determined not by changes in the acceptability of the reason and its connection to the claim alone, but also by one's prior commitment to the claim, and the threshold of acceptability required of the claim. And by the law of total probability (equation 11), the acceptability of a relevant reason changes according to the updated, final probability of a claim.

As an item of potentially good news for informal logicians, our analysis suggests that properly applying the RSA criteria tracks the underlying features of argument cogency just mentioned. But, at best, this indicates a loose affinity between informal and probabilistic approaches to argumentative norms (see Section 5.3). After all, if our analysis is any good, then a probabilistic approach also offers an important corrective to typical informalist applications of these criteria. 


\subsection{Applying the RSA conditions}

Informal logicians who pursue a criterial approach to argument appraisal view argumentative norms as permissive norms. On this approach, the RSA criteria specify individually necessary and jointly sufficient conditions of cogency. Good, or cogent, arguments feature reasons that sufficiently support their conclusions, thereby permitting arguers to accept conclusions on the basis of such reasons.

As we saw in Section 4, a criterial approach readily lends itself to a threshold application, whereby standards of relevance, acceptability, and inferential sufficiency become necessary conditions for inferential update. Since audiences should decline an "invitation to inference" issued in the form of non-cogent arguments, they should not inferentially act upon such arguments. For example, if reasons falling below the acceptability threshold cannot be positively or negatively relevant to a claim-compare Johnson (2000: 342-43) prescribing the application of the acceptability criterion prior to checking an argument for relevance-then unacceptable reasons are impotent in generating support for or against a claim.

However, our analysis suggests that informal logicians commit to important differences concerning the application of the cogency conditions as conditions for inferential update, by comparison to the standards offered by a probabilistic construal of argument cogency. A threshold application for the acceptability of inferential sufficiency of reasons, whereby one ought not to act inferentially upon information that fails to meet some threshold, may lead to mistakes (see Example 1). Relatedly, using the satisfaction of some condition, say acceptability, as a precondition for the testing, or satisfaction, of some other condition, say relevance or sufficiency, can likewise lead to mistakes (see Examples 2, 3).

Generally, then, a criterial approach to the cogency conditions, and thereby to argumentation evaluation, can lead to mistakes. More generally yet, when cogency standards are informally interpreted as permissive norms, they license the acceptance of reasons or permit inferences where these norms are satisfied. On our analysis, by contrast, cogency conditions are not merely entitlement establishing. Rather, they amount to constraints on commitment and its dynamics. That is, satisfying them can be obligatory in view of the probabilistic calculus.

\subsection{Argument appraisal}

Section 2.3 had noted probabilistic analyses for a range of commonplace defeasible arguments and their schemes, including those standardly identified as fallacious and as presumptive. The account of argument cogency offered here complements this work, and contributes to the cohesion and completeness of a probabilistic account of argumentative norms.

Hamblin (1970) argued that fallaciousness is a dialectical rather than a logical or epistemic feature of argument. In this context, the Woods-Walton (Woods \& Walton 2007) approach to the fallacies held that instances of the same argumentative structure could be cogent, or fallacious, depending on context. By contrast, a probabilistic account (e.g., Hahn \& Oaksford, 2007) shows that fallaciousness cannot merely be a dialectical or contextual feature, but that content features - particularly those identified 


\section{DAVID GODDEN AND FRANK ZENKER}

above - are determinative. Importantly, informal logicians came to reject fallacy-based approaches to argument appraisal, on which "[a] good argument will be one that is free of fallacy, and the presence of a fallacy is a prima facie weakness, if not a fatal flaw, in the argument" (Johnson \& Blair, 2002: 369). Instead, Johnson and Blair (2002: 370) argued that fallacies are recognizable and stereotypical failures of the RSA cogency conditions: "[a] fallacy is ... a violation of one or more of the criteria of acceptability, relevance and sufficiency." If so, then some analysis like the one offered here should be welcome. Indeed, if probability theory can account for fallacies, on one hand, but fallacies can also be explained by recourse to the RSA conditions for argument cogency, on the other, then to demonstrate the possibility of a probabilistic analysis of cogency would be an expectable result. In fact, this is just what we have provided.

A similar result holds with argumentation schemes (Walton, Reed, \& Macagno, 2008). Just as the study of the fallacies aims at cataloguing stereotypical ways for arguments to fail, so argumentation schemes seek to provide an inventory of commonplace forms of defeasible, presumptive argument. Informal logicians test and explain the presumptive nature, or probative merit, of instances of such schemes by their satisfaction of the RSA cogency conditions. Similarly with the critical questionsthe usual evaluation tools for schematic arguments. For example, Godden and Walton (2007: 269) claim that: "[ $\mathrm{t}$ ]he function of a critical question is to test a typical or common way in which an argument of a certain schematic type can fail to meet one (or more) of the RSA criteria."

Recently, Hahn and Hornikx (2016), having provided a probabilistic account of three argumentation schemes, conclude that "a Bayesian perspective on the catalogue of argumentation schemes, once systematically applied across the catalogue, will deliver, we think, a comprehensive theory of informal argument" (Hahn \& Hornikx, 2016: 1868f.). Thus, again, if the goodness of schematic arguments is explained-as the informalists claim - by their satisfaction of the RSA cogency conditions, and-as probability theorists claim - by their satisfaction of certain conditions identified in the theory of probability, then theorists should hope to find some probabilistic analysis of the cogency conditions, of the sort offered here. Hahn and Hornikx (2016: 1869) claim that "it is Bayesianism [i.e., probability theory] that carries the normative weight." If so, then the normative success of informal approaches to argument appraisal prior to probabilistic analysis requires some explanation. The explanation our analysis suggests is that the RSA conditions, when applied correctly, track content features of arguments on which their inductive strength depends.

\section{Conclusion}

This paper has provided a probabilistic analysis for the standard informal conditions of argument cogency: acceptability, relevance, and inferential sufficiency (RSA). We have identified content features of defeasible argument on which the cogency conditions depend, namely (i) the change in the acceptability of the reason; (ii) the reason's sensitivity and selectivity to the claim, and (iii) one's prior credence in the claim itself, together with (iv) the contextually determined thresholds of acceptability for reasons and for claims (i.e., inferential sufficiency). 
A probabilistic analysis of the RSA conditions contrast with, and may indeed serve to correct, their orthodox informal understanding and application, for it shows that a threshold application of the orthodox RSA criteria as update-gates can lead to what on a probabilistic construal are mistakes. Moreover, while satisfying the cogency conditions is entitlement establishing, on this analysis cogency is nevertheless an obligatory norm, rather than a permissive one. Finally, our analysis advances the probabilistic approach to argumentation particularly regarding the fallacies and argumentation schemes.

A probabilistic analysis of argument cogency particularly shows how the informal treatment of schemes and fallacies as situated successes or failures to meet the RSA cogency conditions coheres with a probabilistic appraisal of schemes and fallacies. This explains how these two normative theories can in principle, if not in fact, agree, and so contributes to the cohesion and completeness of an account of argumentative norms.

\section{Appendix: Calculations for Example 1 (Section 4.4)}

The law of total probability (equation 11) serves to calculate the initial priors on each of the reasons as follows:

$$
\begin{aligned}
P\left(R_{n}\right) & =P\left(R_{n} \mid C\right) \times P(C)+P\left(R_{n} \mid \sim C\right) \times P(\sim C) \\
& =0.25 \times 0.17+0.15 \times 0.83 \\
& =0.167
\end{aligned}
$$

Using BT (equation 8) to successively update on each reason, $R_{1}$ to $R_{4}$, for the first update:

$$
\begin{aligned}
P_{f}(C) & =\frac{P\left(R_{1} \mid C\right)}{P\left(R_{1}\right)} \times P(C) \\
& =\frac{0.25}{0.167} \times 0.17 \\
& =0.2545
\end{aligned}
$$

Update 1 fails to satisfy equation 28 , since

$$
\frac{P\left(R_{1} \mid C\right)}{P\left(R_{1}\right)}<\frac{t_{S}}{P(C)}=\frac{0.25}{0.167}<\frac{0.5001}{0.17}=1.497<2.492
$$

Given the updated value for $P(C)$, we then recalculate the prior on each remaining reason:

$$
\begin{aligned}
P\left(R_{n}\right) & =P\left(R_{n} \mid C\right) \times P(C)+P\left(R_{n} \mid \sim C\right) \times P(\sim C) \\
& =0.25 \times 0.2545+0.15 \times 0.7455 \\
& =0.1755
\end{aligned}
$$

For the second update, on $R_{2}$, we find:

$$
\begin{aligned}
P_{f}(C) & =\frac{P\left(R_{2} \mid C\right)}{P\left(R_{2}\right)} \times P(C) \\
& =\frac{0.25}{0.1755} \times 0.2545 \\
& =0.3625
\end{aligned}
$$

So also the second update fails to satisfy equation 28 , since

$$
\frac{P\left(R_{2} \mid C\right)}{P\left(R_{2}\right)}<\frac{t_{S}}{P(C)}=\frac{0.25}{0.1755}<\frac{0.5001}{0.12545}=1.425<1.965
$$


Again recalculating the priors on the remaining reasons:

$$
\begin{aligned}
P\left(R_{n}\right) & =P\left(R_{n} \mid C\right) \times P(C)+P\left(R_{n} \mid \sim C\right) \times P(\sim C) \\
& =0.25 \times 0.3625+0.15 \times 0.6375 \\
& =0.1875
\end{aligned}
$$

We find for the third update, on $R_{3}$ :

$$
\begin{aligned}
P_{f}(C) & =\frac{P\left(R_{3} \mid C\right)}{P\left(R_{3}\right)} \times P(C) \\
& =\frac{0.25}{0.1875} \times 0.3625 \\
& =0.4833
\end{aligned}
$$

So that also the third update fails to satisfy equation 28 , since

$\frac{P\left(R_{3} \mid C\right)}{P\left(R_{3}\right)}<\frac{t_{S}}{P(C)}=\frac{0.25}{0.1875}<\frac{0.5001}{0.3625}=1.333<1.379$

Finally recalculating the prior on the remaining reason, $\mathrm{R}_{4}$ :

$$
\begin{aligned}
P\left(R_{n}\right) & =P\left(R_{n} \mid C\right) \times P(C)+P\left(R_{n} \mid \sim C\right) \times P(\sim C) \\
& =0.25 \times 0.4833+0.15 \times 0.6167 \\
& =0.1983
\end{aligned}
$$

For the fourth update we find:

$$
\begin{aligned}
P_{f}(C) & =\frac{P\left(R_{4} \mid C\right)}{P\left(R_{4}\right)} \times P(C) \\
& =\frac{0.25}{0.1983} \times 0.4833 \\
& =0.6093
\end{aligned}
$$

Therefore, had the first three updates already taken place, then a threshold application of sufficiency would permit the fourth update, since

$\frac{P\left(R_{4} \mid C\right)}{P\left(R_{4}\right)} \geq \frac{t_{S}}{P(C)}=\frac{0.25}{0.1983} \geq \frac{0.5001}{0.4833}=1.26 \geq 1.035$

Acknowledgements: We consider this joint work; our names are listed in alphabetical order. For comments that helped improve an earlier version of this paper, we thank Mike Oaksford as well as an anonymous reviewer (the latter particularly on the issue briefly discussed in Section 4.4, footnote 24). A version of this paper was presented at the workshop on Argument Strength hosted by the Research Group for Non-Monotonic Logics and Formal Argumentation at the Institute of Philosophy II, Ruhr-University Bochum, Germany, 30 November-2 December, 2016. We thank that audience for their comments and discussion. Frank Zenker acknowledges a European Union Marie Sklodowska Curie COFUND fellowship (1225/02/03) as well as funding from the Volkswagen Foundation (90 531) and the Ragnar Söderberg Foundation. 


\section{References}

Bayes, T. (1763/1958). An essay towards solving a problem in the doctrine of chances. Philosophical Transactions of the Royal Society of London, 53, 370-418. Reprinted in Biometrika, 45, 296-315.

Blair, J.A. (2011). Informal logic and its early historical development. Studies in Logic, 4, 1-16.

Blair, J.A. (2012). Relevance, acceptability and sufficiency today. In C. Tindale (Ed.), Groundwork in the theory of argumentation (pp. 87-100). Dordrecht: Springer.

Blair, J.A., \& Johnson, R. (1987). The current state of informal logic. Informal Logic, 9, 147-151.

Bradley, S. (2015). Imprecise probabilities. In E.N. Zalta et al. (Eds.), Stanford encyclopedia of philosophy, 2015 edition. Stanford, CA: Center for Study of Language and Information. http://plato.stanford.edu/archives/sum2015/entries/imprecise-probabilities/

Cohen, J.L. (1989). An introduction to the philosophy of induction and probability. Oxford: Oxford UP.

Carnap, R. (1962). The logical foundations of probability, 2nd ed. Chicago: University of Chicago Press.

Corner, A., \& Hahn, U. (2013). Normative theories of argumentation: Are some norms better than others? Synthese, 190, 3579-3610.

Corner, A., Hahn, U., \& Oaksford, M. (2006). The slippery slope argument: Probability, utility and category reappraisal. In R. Sun (Ed.), Proceedings of the 28th Annual Meeting of the Cognitive Science Society, (pp. 1145-1150). Mahwah, NJ: Erlbaum.

Cox, R.T. (1946). Probability, frequency and reasonable expectation. American Journal of Physics, 14, 110.

Cox, R.T. (1961). The algebra of probable inference. Baltimore, MD: Johns Hopkins UP.

Douven, I., \& Schupbach, J.N. (2015). The role of explanatory considerations in updating. Cognition, 142, 299-311.

Eemeren, F.H. van, Garssen, B., Krabbe, E.C.W., Snoeck Henkemans, A.F., Verheij, B., \& Wegemans, J. (2014). Handbook of argumentation theory. Dordrecht: Springer.

Evans, J.S.T.B. (2002). Logic and human reasoning: An assessment of the deduction paradigm. Psychological Bulletin, 128, 978-996.

Fitelson, B. (2001). Studies in Bayesian confirmation theory. Dissertation, University of Wisconsin at Madison. http://fitelson.org/thesis.pdf.

Godden, D. (2010). The importance of belief in argumentation: Belief, commitment and the effective resolution of a difference of opinion. Synthese, 172, 397-414.

Godden, D., \& Walton, D. (2007). Advances in the theory of argumentation schemes and critical questions. Informal Logic, 27, 267-292.

Godden, D., and Zenker F. (2015). Denying antecedents and affirming consequents: The state of the art. Informal Logic, 35, 88-134.

Govier, T. (2010). A practical study of argument, 7th ed. Belmont, CA: Wadsworth, Cengage Learning.

Hahn, U. (2014). The Bayesian boom: good thing or bad?. Frontiers of Psychology, 5, 765. doi: 10.3389/fpsyg.2014.00765

Hahn, U., \& Hornikx, J. (2016). A normative framework for argument quality: Argumentation schemes with a Bayesian foundation. Synthese, 193, 1833-1873.

Hahn, U., \& Oaksford, M. (2006a). A Bayesian approach to informal argument fallacies. Synthese, 152, 207-236.

Hahn, U., \& Oaksford, M. (2006b). A normative theory of argument strength. Informal Logic, 26, 1-24.

Hahn, U., \& Oaksford, M. (2007). The rationality of informal argumentation: A Bayesian approach to reasoning fallacies. Psychological Review, 114, 704-732.

Hahn, U., Oaksford, M., \& Bayindir, H. (2005). How convinced should we be by negative evidence? In B. Bara, L. Barsalou, \& M. Bucciarelli (Eds.), Proceedings of the 27th Annual Conference of the Cognitive Science Society (pp. 887-892). Mahwah, N.J.: Lawrence Erlbaum Associates.

Hahn, U., Oaksford, M., \& Corner, A. (2005). Circular arguments, begging the question and the formalization of argument strength. In A. Russell, T. Honkela, K. Lagus, \& M. Pöllä, (Eds.), Proceedings of AMKLC'05, International Symposium on Adaptive Models of Knowledge, Language and Cognition, (pp. 34-40). Espoo, Finland: Helsinki University of Technology.

Hajek, A. (2008). Dutch book arguments. In: Anand, P., Pattanaik, P., and Puppe, C. Eds.), The Oxford handbook of rational and social choice (pp. 173-195). Oxford. Oxford UP.

Hamblin, C. (1970). Fallacies. London: Methuen.

Harris, A.J.L., Hahn, U., Madsen, J.K., \& Hsu, A. (2015). The appeal to expert opinion: Quantitative support for a Bayesian network approach. Cognitive Science, DOI: 10.1111/cogs.12276. 


\section{DAVID GODDEN AND FRANK ZENKER}

Hertwig, R., Ortmann, A., \& Gigerenzer, G. (1997). Deductive competence: A desert devoid of content and context. Current Psychology of Cognition, 16, 102-107.

Howson, C., \& Urbach, P. (2006). Scientific reasoning: The Bayesian approach, 3rd ed. La Salle, IL: Open Court.

Ikuenobe, P. (2004). On the theoretical unification and nature of the fallacies. Argumentation, 18, 189211.

Jeffrey, R. (1983). The logic of decision, 2nd ed. Chicago: University of Chicago Press.

Johnson, R. (2000). Manifest rationality: A pragmatic theory of argument. Mahwah, NJ: Lawrence Earbaum.

Johnson, R. H. (2006). Making sense of informal logic. Informal Logic, 26, 231-258.

Johnson, R. (2011). Informal logic and deductivism. Studies in Logic, 4, 17-37.

Johnson, R., \& Blair, JA. (2002.) Informal logic and the reconfiguration of logic. In D. Gabbay, R. Johnson, H. Ohlbach, \& J. Woods (Eds.), Handbook of the logic of argument and inference: Turn towards the practical (pp. 340-396). Amsterdam and New York: Elsevier.

Johnson, R., \& Blair, J.A. (2006). Logical self defense, 3rd ed. New York: International Debate Education Association. (First edition 1977, Toronto: McGraw-Hill Ryerson.)

Joyce, J. (2009). Bayes' theorem. In E.N. Zalta et al. (Eds.), Stanford encyclopedia of philosophy, 2009 edition (pp. 1-47). Stanford, CA: Center for Study of Language and Information. http://plato.stanford.edu/archives/spr2009/entries/bayes-theorem/

Kolmogorov, A.N. (1933). Grundbegriffe der Wahrscheinlichkeitrechnung, Ergebnisse Der Mathematik. Berlin: Springer (translated as: 1950. Foundations of Probability, New York: Chelsea Publishing Company).

Korb, K. (2004). Bayesian informal logic and fallacy. Informal Logic, 24, 41-70.

Oaksford, M., \& Hahn, U. (2004). A Bayesian approach to the argument from ignorance. Canadian Journal for Experimental Psychology, 58, 75-85.

Pfeifer, N. (2013). On argument strength. In F. Zenker (Ed.), Bayesian argumentation: The practical side of probability (pp. 185-193). Dordrecht: Springer.

Pinto, R.C. (2001). Argument, inference and dialectic. Dordrecht: Kluwer.

Powers, L.H. (1995). The one fallacy theory. Informal Logic, 17(2): 303-314.

Ramsey, F. (1926/1931). Truth and probability. In R. Braithwaite (Ed.), The foundations of mathematics and other essays (pp. 156-198). London: Routledge \& Kegan Paul.

Spohn, W. (2012). The laws of belief: Ranking functions and their applications. Oxford: Oxford UP.

Strevens, M. (2012). Notes on Bayesian confirmation theory. http://www.strevens.org/bct/

Talbot, W. (2011). Bayesian epistemology. In E.N. Zalta et al. (Eds.), Stanford encyclopedia of philosophy, 2011 edition (pp. 1-34). Stanford, CA: Center for Study of Language and Information. http://plato.stanford.edu/archives/sum2011/entries/epistemology-bayesian/

Vorobej, M. (2006). A theory of argument. New York: Cambridge UP.

Walton, D., \& Gordon, T. (2015). Formalizing informal logic. Informal Logic, 35, 508-538.

Walton, D., Reed, C., \& Macagno, F. (2008). Argumentation schemes. Cambridge: Cambridge UP.

Woods, J. (2000). How philosophical is informal logic? Informal Logic, 20, 139-167.

Woods, J., \& Walton, D. (2007). Fallacies: Selected papers 1972-1982. London: College Publications.

Zenker, F. (2013). Bayesian argumentation: The practical side of probability. In F. Zenker (Ed.), Bayesian argumentation: The practical side of probability (pp. 1-11). Dordrecht: Springer.

Zenker, F. (2016). The polysemy of 'fallacy' — or 'bias', for that matter. In: Bondy, P., and Benaquista, L. (eds). Argumentation, Objectivity and Bias (Proceedings of the $11^{\text {th }}$ Conference of the Ontario Society for the Study of Argumentation, 18-21 May, 2016) (pp. 1-14). Windsor, ON: OSSA. http://scholar.uwindsor.ca/ossaarchive/OSSA11/ 